\title{
Estudio prospectivo para la enseñanza superior virtual al 2030
}

\author{
Recibido: 18/11/11 Aceptado: 26/06/12
}

\author{
Jorge IncheMitma ${ }^{1}$ \\ Alfonso Ramón Chung Pinzás ${ }^{2}$
}

\begin{abstract}
RESUMEN
El presente artículo intenta respondar la siguiente pregunta: ¿cómo formular un escenario futurible para la educación superior virtual al 2030?; para ello se aplicó las herramientas de la prospectiva estratégica de Michel Godet: Análisis estructural (MICMAC), matriz de actores (MACTOR) y análisis de escenarios (SMIC). Los resultados muestran dos escenarios de mayor probabilidad, los cuales permitirán establecer acciones estratégicas para construir un futuro posible y deseable para la educación virtual en el Perú al 2030.
\end{abstract}

Palabras claves: prospectiva estratégica, educación virtual, futurible, internet, docencia.

\section{PROSPECTIVE STUDY TO THE SUPERIOR VIRTUAL EDUCATION AT 2030}

\section{ABSTRACT}

This paper analyzes the next problematic question: how to formulate a potential scenario to the virtual superior education at 2030?; for this, will use prospective tools such as structural analyze, actors and objectives matrix (MACTOR) and the SMIC method. This research will allow to the universities adequate their plans and program in order to develop the desired future.

Keywords: strategic prospective, virtual education, potential, internet, teaching.

\section{INTRODUCCIÓN}

El análisis de los escenarios futuribles es de gran importancia en cualquier organización, debido a que le permite a los actores ser partícipes en la construcción del futuro deseado [3]. El presente estudio se justifica en todo escenario futurible permitirá a las instituciones de educación superior contar con un rumbo para poder planificar sus actividades a futuro, asimismo, permitirá introducir el pensamiento prospectivo en las actividades académico universitarias. Entre los beneficiarios se encuentran las instituciones universitarias, los estudiantes, docentes autoridades etc., pero también se espera mostrar en forma resumida, una metodología prospectiva que pueda ser replicada en otras instituciones.

Los objetivos del estudio realizado fueron los siguientes:

a. Identificar los elementos estructurales para la definición de variables estratégicas de la prospectiva.

b. Determinar el rol de actores claves.

c. Determinar los escenarios probables y el núcleo tendencial.

d. Elaborar el escenario apuesta.

Como antecedentes metodológicos se pueden citar dos ejercicios anteriores realizados por el equipo de trabajo: "Prospectiva en el cluster de muebles de Villa el Salvador" (publicado en la revista Industrial Data Vol. 9 № 1, año 2006) y "Prospectiva estratégica al sector textil en San Juan de Lurigancho" (también

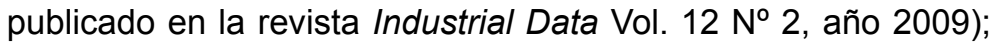
si bien en ambos casos la aplicación fue en escenarios industriales, en este caso, se analiza un entorno distinto como es la educación superior virtual.

La metodología aplicada corresponde a la escuela francesa de la prospectiva estratégica, de la cual el Dr. Michel Godet es quizás el más reconocido de sus representantes; su libro electrónico La caja de herramientas de la prospectiva estratégica es un material de lectura obligatoria para quien desee profundizar en este tema. 


\section{Marco teórico}

El sistema educativo, no solo latinoamericano, sino mundial, se encuentra fuertemente impactado por el fenómeno de la globalización y su nuevo empeño "civilizatorio": la sociedad del conocimiento. En la región latinoamericana, esta llega para aumentar la complejidad de los sistemas educativos nacionales, los cuales ahora no solo enfrentan rezagos múltiples del siglo $\mathrm{XX}$, sino tendrán que incorporar los nuevos retos que implica la globalización. La región se encuentra atrasada y rezagada en cumplir con los fines y las metas de ofrecer educación para todos y reducir la exclusión e inequidad social; y ahora afronta la necesidad de garantizar la calidad y la actualización permanente de los conocimientos, capacidades y valores.

Brunner (2000) propone tres estrategias para capitalizar el uso de las TIC en el esfuerzo educativo regional: 1) instituir el aprendizaje a lo largo de toda la vida que incluya no solo un saber hacer sino la capacidad para convivir en diversos mundos de vida, sin que eso signifique necesariamente la descolarización; 2) universalizar el uso de las TIC por medio de una conectividad total que haga sustentable la interactividad y 3) institucionalizar redes de tal manera que sea posible una educación abierta, flexible, integrada y cercana a las comunidades [1].

Asimismo, Facundo (2002) plantea los siguientes puntos: 1) una política conjunta (regional) para mejorar la conectividad (módems de banda ancha, satélites y fibraóptica), así como abaratar costos de equipo y tarifas de conexión y subsidio a pobres que permitan el equipamiento tecnológico de las aulas y un uso común entre docentes y alumnos; 2) crear una cultura de apertura hacia las TIC, donde se capacite a todos los miembros de la comunidad y se aprenda a diseñar y gestionar programas virtuales; 3) establecer convenios y conformar redes de cooperación real entre las instituciones de la región; 4) formar equipos regionales estables y multidisciplinarios de investigación que desarrollen proyectos para construir políticas públicas de educación virtual [2].

Schwartzmann (2001) predice que las innovaciones pedagógicas lograrán una mayor descentralización organizacional y de gestión de la educación, incluyendo una mayor participación de la comunidad, de las bases académicas y estudiantiles; asimismo, se usarán intensamente métodos cuantitativos de evaluación del desempeño de profesores y alumnos para orientar políticas públicas lo cual devendrá en una mejoría relativa en la educación básica y secundaria [10].

La educación virtual constituye un importante aporte para la difusión del conocimiento, al respecto
Miklos y Arroyo (2008) sostienen que los puntos más resaltantes de un escenario futurible deberían ser los siguientes [5]:

a. Expansión de las nuevas tecnologías.

b. Socialización democrática de las tecnologías.

c. Modelos educativos flexibles y pertinentes apoyados en las TIC.

d. Reconfiguración lenta de las instituciones.

e. Educación virtual como mecanismo para ampliar la cobertura y el acceso a cierta calidad en el aprendizaje.

f. "Lo futurible no tiene que cumplirse, es un orientador, brújula que permite pensar en lo deseable".

Como se puede apreciar, existen dos constates fundamentales entre las características mencionadas anteriormente, tecnologías y modelos educativos. En efecto, las tecnologías hoy en día están creciendo en cuanto a diversidad, capacidad etc., por lo tanto, el proceso educativo debe verse impactado por este hecho; cambiando dialécticamente muchas de sus concepciones primigenias.

Por otro lado, Canepa (2004) y Camones (2010) presentan los siguientes puntos claves para el caso peruano [6]:

a. Las modalidades tradicionales tienen dificultades para el acceso y concurrencia de la población a un centro educativo.

b. La educación virtual permite flexibilizar horarios y alcanzar mayor audiencia.

c. En la actualidad, la mayoría de universidades dispone de programas semivirtuales.

d. Muy pocas universidades tienen programas totalmente virtuales.

e. Genera dependencia y fragmentación social.

f. De cada cuatro peruanos uno es usuario de internet.

g. Tendencia de las universidades a utilizar la educación virtual como complemento de las clases presenciales.

Según este estudio se puede apreciar que en el caso peruano, aún se está implementando esta modalidad, mayormente el uso es como complemento a las clases (generalmente mediante aulas virtuales y como repositorio); sin embargo, todavía no se puede hablar de universidades totalmente virtuales ya que son muy pocos los casos. 


\section{METODOLOGÍA}

Se fijó como horizonte temporal para el análisis: 20 años. Fijar un horizonte de largo plazo permite a los analistas desprenderse de la coyuntura actual (presentismo), romper la alta densidad de la coyuntura y alejarse de su determinismo. Se conformó un grupo de 15 expertos que se reunió en talleres de prospectiva bajo la metodología de la escuela francesa de Michel Godet [4].

El camino desde la identificación de variables hasta la búsqueda de escenarios futuribles, tuvo el siguiente recorrido:

Figura 1: Metodología de trabajo

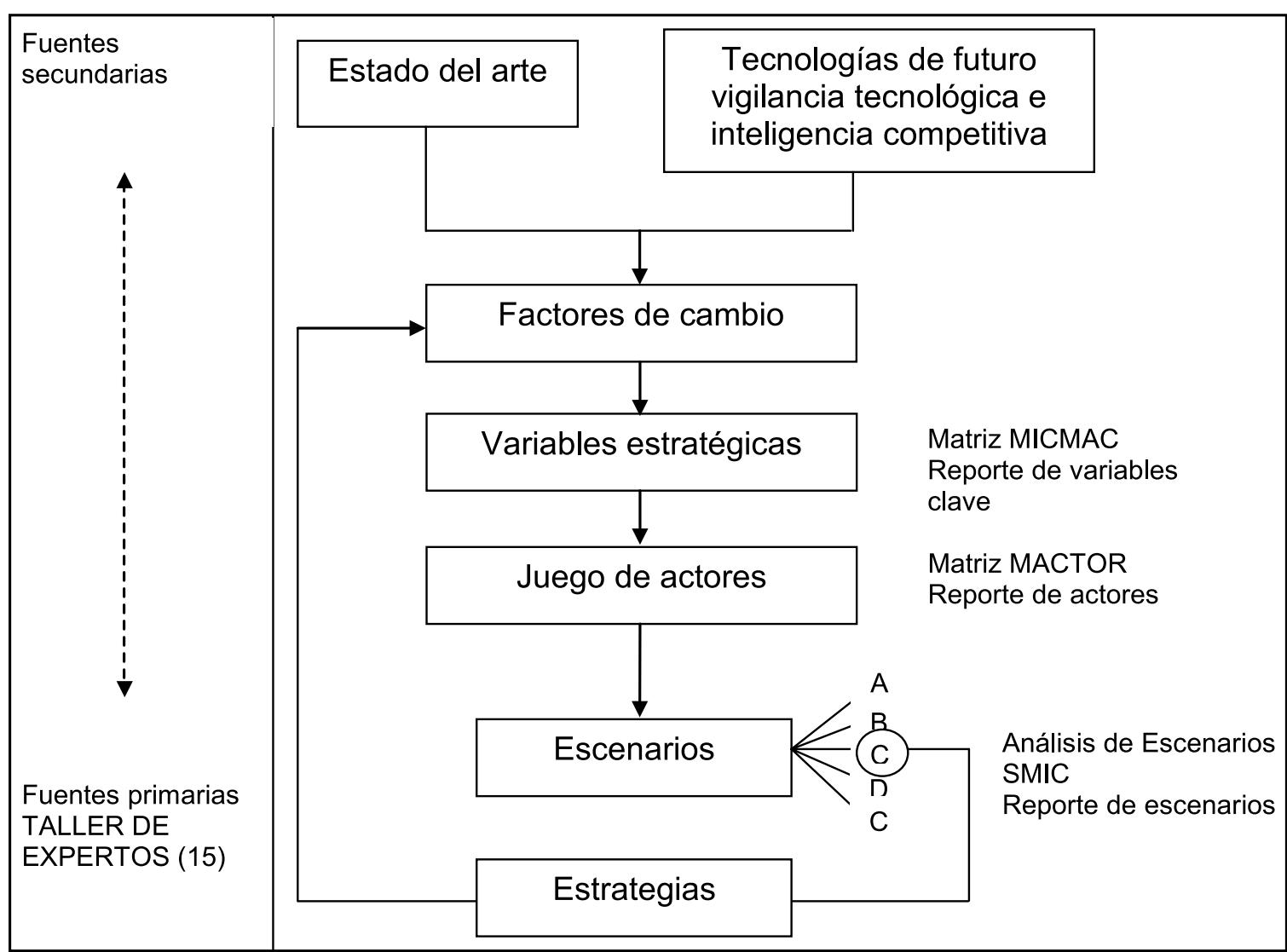

\section{RESULTADOS DE LA INVESTIGACIÓN}

Como se mencionó anteriormente, la investigación se realizó bajo el marco de la prospectiva estratégica; las herramientas utilizadas fueron las siguientes:

a. Análisis estructural para determinar las variables claves [7].

b. Matriz de actores (MACTOR), a fin de identificar los principales actores y su posición en el sistema de estudio [8].
C. SMIC para identificar los escenarios más probables e identificar el escenario apuesta [9].

Como expertos se han designado a docentes universitarios, prospectivistas en educación superior y analistas en general; para la identificación de variables del sistema se utilizó una encuesta en línea (internet) cuyos resultados mostraron 43 variables en el ámbito, político, social, económico y tecnológico; las cuales se listan a continuación: 


\section{Cuadro 1: Variables identificadas}

\begin{tabular}{|l|l|}
\hline VAR 1 & Crecimiento económico \\
\hline VAR 2 & Tiempo de transporte al aula presencial \\
\hline VAR 3 & Avance de la tecnología \\
\hline VAR 4 & Inflación \\
\hline VAR 5 & Nivel de empleo \\
\hline VAR 6 & Globalización \\
\hline VAR 7 & Horas de clases virtuales \\
\hline VAR 8 & Presupuesto estatal \\
\hline VAR 9 & Nivel de ingresos \\
\hline VAR 10 & Costo de la enseñanza virtual \\
\hline VAR 11 & Diversificación de mercado \\
\hline VAR 12 & Diversificación de trabajo \\
\hline VAR 13 & Indicadores de productividad laboral \\
\hline VAR 14 & Costo para el desarrollo de tutoriales y clases virtuales \\
\hline VAR 15 & Costos de infraestructura tecnológica \\
\hline VAR 16 & Crisis económica \\
\hline VAR 17 & Estabilidad social \\
\hline VAR 18 & Idioma y cultura de poblaciones objetivo \\
\hline VAR 19 & Mapa de pobreza \\
\hline VAR 20 & Becas \\
\hline VAR 21 & Emprendedurismo \\
\hline VAR 22 & Reconocimiento laboral de los estudios virtuales \\
\hline VAR 23 & Cobertura de servicios virtuales \\
\hline VAR 24 & Facilidad de uso de sistemas virtuales \\
\hline
\end{tabular}

\begin{tabular}{|l|l|}
\hline VAR 25 & Moda \\
\hline VAR 26 & Perspectivas laborales \\
\hline VAR 27 & Reconocimiento legal de los estudios virtuales \\
\hline VAR 28 & Ley universitaria \\
\hline VAR 29 & Política educativa \\
\hline VAR 30 & Involucramiento de congresistas y ministros \\
\hline VAR 31 & Nivel de uso de las TIC \\
\hline VAR 32 & Redes sociales \\
\hline VAR 33 & Elección del tipo de tecnología (libre o propietaria) \\
\hline VAR 34 & Capacitación \\
\hline VAR 35 & Biblioteca virtual \\
\hline VAR 36 & Motivación \\
\hline VAR 37 & Apoyo del gobierno central y de instituciones privadas \\
\hline VAR 38 & Adaptación de los planes de estudios \\
\hline VAR 39 & Competencia de señales de internet comerciales \\
\hline VAR 40 & Obsolescencia tecnológica \\
\hline VAR 41 & Proliferación de aulas virtuales, blackboards \\
\hline VAR 42 & Tipos de conocimiento necesarios \\
\hline VAR 43 & Metodología \\
\hline
\end{tabular}

Luego de aplicar el análisis estructural (matrices de impacto directo e indirecto) se pudo posicionar las variables según su naturaleza, tal y como se muestran en la Figura 2.

Figura 2. Ubicación de las variables según su naturaleza

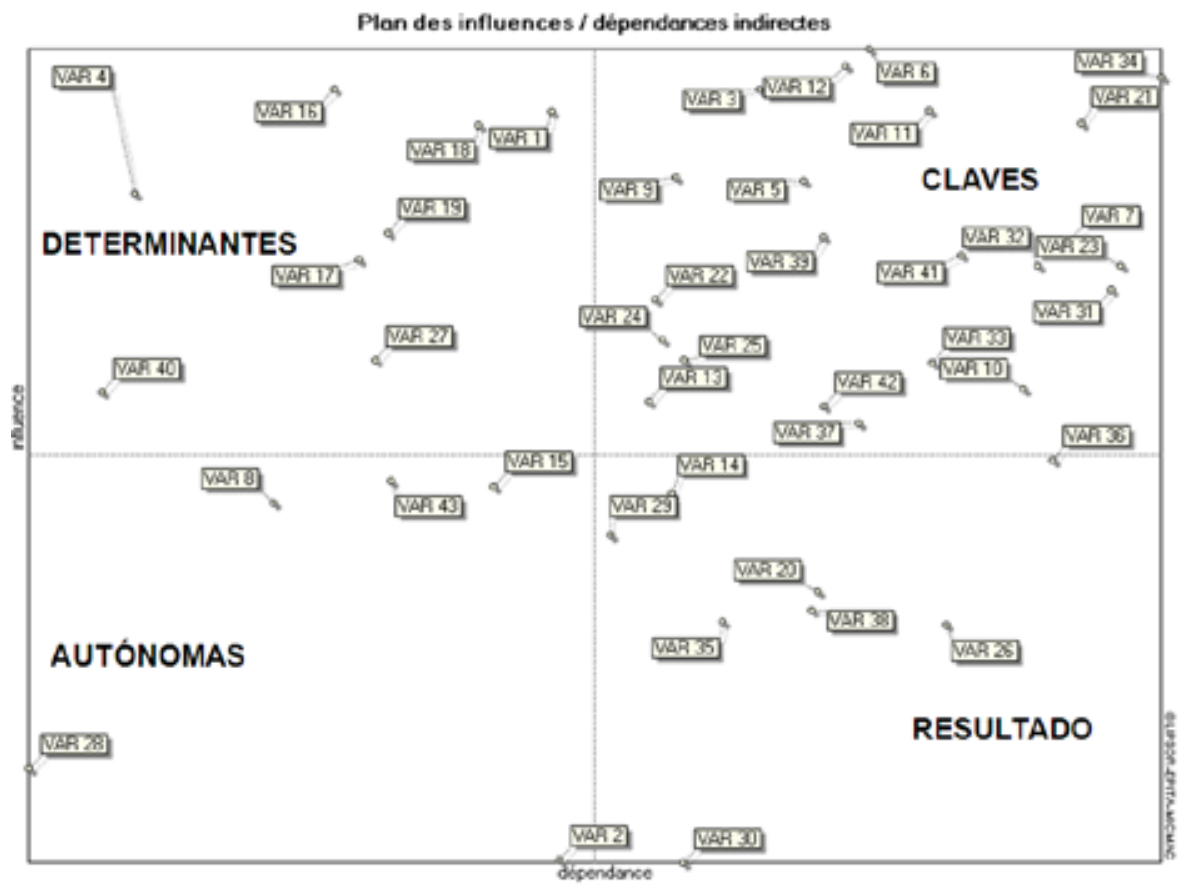

Cómo se puede ver en la figura anterior, existen 22 variables claves (sector superior derecho), es decir, aquellas que tienen un mayor nivel de dependencia e influencia por lo tanto son las más importantes en el sistema; el Cuadro 2 muestra dichas variables con sus respectivos puntajes. 
Cuadro 2: Variables claves

\begin{tabular}{|c|c|c|}
\hline Orden & & Variables clave \\
\hline 1 & VAR 34 & Capacitación \\
\hline 2 & VAR 21 & Emprendedurismo \\
\hline 3 & VAR 6 & Globalización \\
\hline 4 & VAR 11 & Diversificación de mercado \\
\hline 5 & VAR 12 & Diversificación del trabajo \\
\hline 6 & VAR 23 & Cobertura de servicios virtuales \\
\hline 7 & VAR 7 & Horas de clases virtuales \\
\hline 8 & VAR 31 & Nivel de uso de las TIC \\
\hline 9 & VAR 32 & Redes sociales \\
\hline 10 & VAR 3 & Avance de la tecnología \\
\hline 11 & VAR 41 & Proliferación de aulas virtuales, blackboards \\
\hline 12 & VAR 5 & Nivel de empleo \\
\hline 13 & VAR 39 & Competencia de señales de internet comerciales \\
\hline 14 & VAR 10 & Costo de la enseñanza virtual \\
\hline 15 & VAR 9 & Nivel de ingresos \\
\hline 16 & VAR 33 & Elección del tipo de tecnología (libre o propietaria) \\
\hline 17 & VAR 37 & Apoyo del gobierno central y de instituciones privadas \\
\hline 18 & VAR 42 & Tipos de conocimiento necesarios \\
\hline 19 & VAR 22 & Reconocimiento laboral de los estudios virtuales \\
\hline 20 & VAR 24 & Facilidad de uso de sistemas virtuales \\
\hline 21 & VAR 25 & Moda \\
\hline 22 & VAR 13 & Indicadores de productividad laboral \\
\hline
\end{tabular}

Posteriormente se aplicó el análisis MACTOR mediante la matriz de actores por actores; la lista de actores identificados por los expertos es la siguiente (véase cuadro 3 ).
Cuadro 3: Actores identificados

\begin{tabular}{|l|l|}
\hline \multicolumn{1}{|c|}{ Clave } & \multicolumn{1}{|c|}{ Actor } \\
\hline ACT 1 & Gobierno central \\
\hline ACT 2 & Docentes \\
\hline ACT 3 & Alumnos \\
\hline ACT 4 & Empresas privadas \\
\hline ACT 5 & Universidades \\
\hline ACT 6 & Tutores de las cátedras y asignaturas \\
\hline ACT 7 & Acreditadores \\
\hline ACT 8 & Arquitectos del Sistema de Información \\
\hline ACT 9 & Colegios profesionales \\
\hline ACT 10 & Políticos \\
\hline ACT 11 & Metodólogos \\
\hline
\end{tabular}

La ubicación en el plano $\mathrm{X}, \mathrm{Y}$ así como su posición frente al sistema está dada por la Figura 3 que es resultado de las matrices anteriormente indicadas.

Esta información será útil posteriormente cuando se inicien las estrategias para la construcción del escenario apuesta, ya que se puede considerar la naturaleza que tiene el actor y cómo negociar con él.

Regresando a las variables claves, Godet (2000) recomienda considerar cinco variables claves para la formulación de las hipótesis respectivas, cuyas combinaciones darán lugar a los posibles escenarios futuribles; el Cuadro 4 muestra las cinco variables con sus respectivas hipótesis.

Figura 3. Ubicación de los actores según su naturaleza

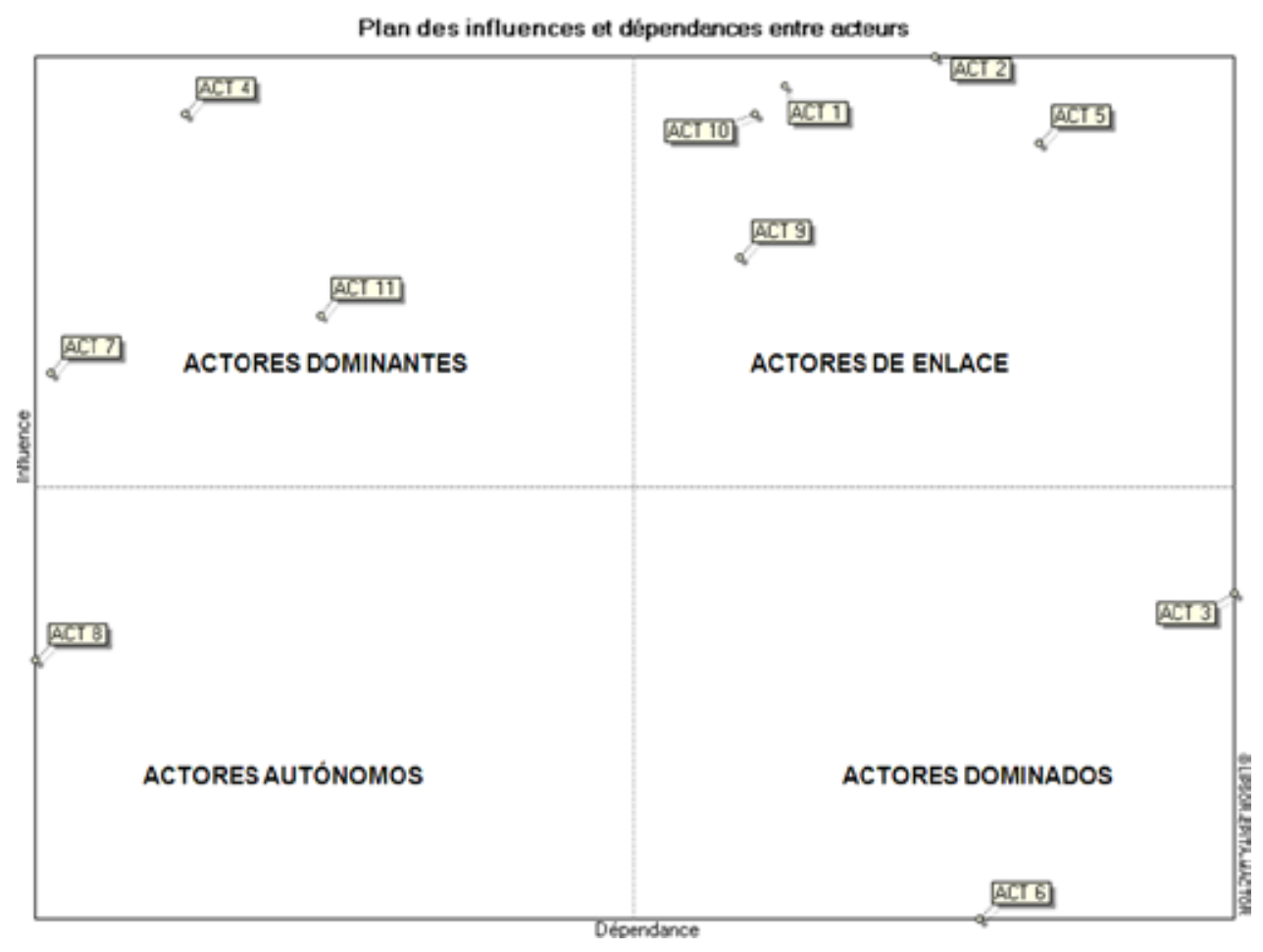


Cuadro 4: Variables e hipótesis

\begin{tabular}{|c|c|c|}
\hline Clave & Nombre & Hipótesis \\
\hline VAR 34 & Capacitación & $\begin{array}{l}\text { Se ha logrado un nivel de capacitación bastante elevado a consecuencia de los programas de educación superior virtual, no } \\
\text { solo en áreas temáticas específicas, sino también en la importancia de este sistema; de hecho, gran parte de la población } \\
\text { es consciente de los beneficios de la educación virtual, además conoce acerca del uso de sus instrumentos. Todo esto ha } \\
\text { impactado positivamente en el nivel de desarrollo, el avance de la tecnología, el tipo de trabajos realizados, así como el nivel } \\
\text { de integración del país y la matriz de las actividades económicas que se desarrollen en el Perú. }\end{array}$ \\
\hline VAR 21 & Emprendedurismo & $\begin{array}{l}\text { El nivel de emprendedurismo en el país ha aumentado gracias al impulso de la educación virtual superior, asimismo los } \\
\text { programas virtuales contienen componentes relacionados al emprededurismo, los cuales han sido ampliamente difundidos } \\
\text { en diversas partes del Perú y además han contribuido a generar mayor valor agregado a la producción de distintos bienes } \\
\text { y servicios, mejorando los ingresos y la calidad de vida. }\end{array}$ \\
\hline VAR 6 & Globalización & $\begin{array}{l}\text { Los programas de educación superior virtual, han logrado globalizar los conocimientos en diversas temáticas de interés a } \\
\text { muchas más personas, descentralizando la enseñanza y logrando mayores beneficios a los participantes, trayendo como } \\
\text { consecuencia una mayor integración de las personas con un enfoque de diversidad, inclusión y equidad. }\end{array}$ \\
\hline VAR 11 & Diversificación de mercado & $\begin{array}{l}\text { El mercado para la enseñanza superior virtual se ha diversificado, esto debido a la facilidad de acceso y a los bajos costos, } \\
\text { sumado al uso de herramientas que facilitan el aprendizaje en diversas ramas del conocimiento y a su carácter propiamente } \\
\text { innovador en cuanto a la metodología empleada. }\end{array}$ \\
\hline VAR 12 & Diversificación del trabajo & $\begin{array}{l}\text { Debido a la masificación del uso de los programas virtuales de enseñanza superior, se ha logrado diversificar las fuentes de } \\
\text { trabajo, atendiendo muchas áreas o nichos que no se habían considerado anteriormente y también creando nuevas fuentes } \\
\text { de trabajo, lo cual a contribuido al bienestar de la población en general. }\end{array}$ \\
\hline
\end{tabular}

Luego de la aplicación del método SMIC, se tienen seis hipótesis en el núcleo tendencial (82\%), de las cuales las dos primeras tienen una fuerte probabilidad de ocurrencia (cuadro 5).

Cuadro 5: Escenarios y probabilidades de ocurrencia

\begin{tabular}{|c|c|c|c|}
\hline \multirow{2}{*}{ Escenario } & \multicolumn{2}{|c|}{ Probabilidad } & \multirow{2}{*}{ Porcentaje } \\
\cline { 2 - 3 } & Individual & Acumulada & \\
\hline $01-11111$ & 0.348 & 0.348 & $35 \%$ \\
\hline $32-00000$ & 0.246 & 0.594 & $59 \%$ \\
\hline $05-11011$ & 0.066 & 0.660 & $66 \%$ \\
\hline $03-11101$ & 0.057 & 0.717 & $72 \%$ \\
\hline $02-11110$ & 0.053 & 0.770 & $77 \%$ \\
\hline $10-10110$ & 0.049 & 0.819 & $82 \%$ \\
\hline $16-10000$ & 0.045 & 0.864 & $86 \%$ \\
\hline $09-10111$ & 0.038 & 0.902 & $90 \%$ \\
\hline $08-11000$ & 0.037 & 0.939 & $94 \%$ \\
\hline $21-01011$ & 0.020 & 0.959 & $96 \%$ \\
\hline $14-10010$ & 0.015 & 0.974 & $97 \%$ \\
\hline $17-01111$ & 0.014 & 0.988 & $99 \%$ \\
\hline $30-00010$ & 0.008 & 0.996 & $100 \%$ \\
\hline $04-11100$ & 0.003 & 0.999 & $100 \%$ \\
\hline $29-00011$ & 0.003 & 1.002 & $100 \%$ \\
\hline $07-11001$ & 0.001 & 1.003 & $100 \%$ \\
\hline $06-11010$ & 0.000 & 1.003 & $100 \%$ \\
\hline $11-10101$ & 0.000 & 1.003 & $100 \%$ \\
\hline $12-10100$ & 0.000 & 1.003 & $100 \%$ \\
\hline $13-10011$ & 0.000 & 1.003 & $100 \%$ \\
\hline
\end{tabular}

\begin{tabular}{|l|l|l|l|}
\hline $15-10001$ & 0.000 & 1.003 & $100 \%$ \\
\hline $18-01110$ & 0.000 & 1.003 & $100 \%$ \\
\hline $19-01101$ & 0.000 & 1.003 & $100 \%$ \\
\hline $20-01100$ & 0.000 & 1.003 & $100 \%$ \\
\hline $22-01010$ & 0.000 & 1.003 & $100 \%$ \\
\hline $23-01001$ & 0.000 & 1.003 & $100 \%$ \\
\hline $24-01000$ & 0.000 & 1.003 & $100 \%$ \\
\hline $25-00111$ & 0.000 & 1.003 & $100 \%$ \\
\hline $26-00110$ & 0.000 & 1.003 & $100 \%$ \\
\hline $27-00101$ & 0.000 & 1.003 & $100 \%$ \\
\hline $28-00100$ & 0.000 & 1.003 & $100 \%$ \\
\hline $31-00001$ & 0.000 & 1.003 & $100 \%$ \\
\hline
\end{tabular}

Fuente: Equipo de trabajo

El cuadro anterior ha sido ordenado de mayor a menor probabilidad; asimismo, para un mejor entendimiento de la nomenclatura empleada, se analiza el siguiente ejemplo:

05 - 11011 es el escenario 5, de los cuales solo la hipótesis 3 (la que tiene el valor de 0 ) no se cumple, el resto de hipótesis del Cuadro 4 se realizan (ya que tienen valor 1 ).

Como se puede apreciar en el Cuadro 5, el escenario de mayor probabilidad de ocurrencia es 01$11111(0,348$ o $34,8 \%)$, es decir, el escenario opti- 
mista; sin embargo, el escenario pesimista está en segundo lugar, es decir $32-00000$.

\section{CONCLUSIONES}

1. Un escenario optimista es posible de construir en el sector estudiado, siempre y cuando se tomen las acciones respectivas para tal caso.

2. Sin embargo, de no realizar ningún cambio, el escenario negativo tiene una alta probabilidad de ocurrencia (59\%).

3. Los actores tienen un rol de gran importancia para la construcción del escenario futuro.

4. La implementación de un sistema de educación superior virtual es aún una tarea pendiente en las universidades peruanas.

\section{REFERENCIAS BIBLIOGRÁFICAS}

[1] Brunner, J. (2000). "Globalización y el futuro de la educación: tendencias, desafíos, estrategias". Documento de trabajo presentado en el Seminario sobre prospectivas de la educación en la región de américa latina y el caribe, organizado por la Oficina Regional de Educación de la UNESCO (Santiago de Chile, 23/25 de agosto de 2000), www.brunner. cl.com.
[2] Facundo, A. H. (2002). "Educación virtual en América Latina y El Caribe: características y tendencias", UNESCO-IESALC, Bogotá.

[3] Godet, M. (2000). Caja de herramientas de la prospectiva estratégica. Edición Digital, LIPSOR.

[4] Godet, M. (2009). Manual de prospectiva estratégica. Edición Digital, LIPSOR.

[5] Miklos T. y Arroyo M. (2008). "Una visión prospectiva de la educación a distancia en América Latina", Universidades, Vol. 58, Nº 37: 49-67.

[6] Canepa C. (2004). "Un modelo peruano de educación virtual: el caso de la universidad virtual de la UNMSM", Revista de Investigación y Sistemas de Información, Vol. 1, № 1 : 87-92.

[7] Lipsor (2005). MICMAC. Se poser les bonnes questions et identifier les variables clés, http:// www.3ie.org/lipsor/micmac.htm (visitado el 24-08-11)

[8] Lipsor (2005). Analyser les stratégies d'acteurs, http://www.3ie.org/lipsor/mactor.htm (visitado el 24-08-11)

[9] Lipsor (2005). SMIC-PROB-EXPERT, Impact scroisés probabilistes, http://www.3ie.org/lipsor/mactor.htm (visitado el 24-08-11).

[10] Schwartzmann, S. (2001): "El futuro de la educación en América Latina y El Caribe" en UNESCO / OREALC (ED-01 / PROMEDLACVII / REF.2). 